\title{
Dirofilaria Immitis: Genotyping European Clinical Samples and US Laboratory Isolates at Single Nucleotide Polymorphic (SNP) Sites Associated With Macrocyclic Lactone Susceptibility and Resistance
}

\section{Emily Curry}

McGill University Institute of Parasitology

\section{Donato Traversa}

University of Teramo: Universita degli Studi di Teramo

Elena Cárreton

University of Las Palmas de Gran Canaria: Universidad de las Palmas de Gran Canaria

\section{Laura Kramer}

University of Parma Department of Veterinary Health Sciences: Universita degli Studi di Parma

Dipartimento di Scienze Medico-Veterinarie

Heinz Sager

Elanco International Inc: Elanco Animal Health Inc

\section{Lisa Young}

Elanco Animal Health Inc

Roger K Prichard ( $\square$ roger.prichard@mcgill.ca )

McGill University

\section{Research Article}

Keywords: Dirofilaria immitis, Dirofilariosis, Macrocyclic Lactones, Suceptibility, Resistance, Molecular Markers, Single Nucleotide Polymorphism

Posted Date: January 24th, 2022

DOI: https://doi.org/10.21203/rs.3.rs-1258381/v1

License: (c) (i) This work is licensed under a Creative Commons Attribution 4.0 International License.

Read Full License 


\section{Abstract}

Background: Dirofilaria immitis is a parasitic filarial nematode. It is the causative agent of dirofilariosis, a potentially fatal pulmonary infection which primarily infects canids and felines. dirofilariosis infections are primarily controlled with a prophylactic macrocyclic lactone $(M L)$ regimen. Recent evidence has confirmed the development of ML-resistant isolates in the US which are genetically distinct from wild-type populations. Previous research clinically validated 9 single nucleotide polymorphism (SNP) molecular markers associated with these ML resistance phenotypes isolated from the USA.

Methods: In this study, three $D$. immitis US laboratory-maintained isolates: two susceptible isolates, Berkeley, and Georgia II, one resistant isolate, WildCat; and eleven European D. immitis clinical samples, from Italy, Spain, and Hungary were analyzed. The samples tested were fresh microfilaria ( $\mathrm{mf}$ ) in blood or adult female worms shipped in ethanol and rehydrated in phosphate buffered saline (PBS). After DNA extraction, each sample underwent MiSeq sequencing of regions encompassing the 9 SNP sites previously associated with macrocyclic lactone susceptibility and resistance. The nucleotide frequencies of the 9 SNP sites were analyzed and the pairwise fixation index $\left(\mathrm{F}_{\mathrm{ST}}\right)$ of the top 2 SNP molecular markers were calculated to estimate the probability of identity with known susceptible and resistant isolates.

Results: In the three laboratory-maintained US D. immitis isolates Berkeley had a 2-SNP pairwise $\mathrm{F}_{\mathrm{ST}}$ of 0.00 , Georgia II had a 2-SNP pairwise $F_{S T}$ of 0.07 indicating two ML-susceptible genotypes. WildCat had a 2-SNP pairwise $F_{S T}$ of 0.33 indicating a ML-resistant genotype. The genotype analysis of the European clinical samples showed that all eleven had 2-SNP pairwise $F_{S T}$ of 0.00 , which indicates their genotypes, at these SNP sites, are consistent with ML susceptibility.

Conclusions: The results of the current study provides a genotypic analysis of European clinical samples with US SNP molecular markers associated with ML susceptibility and resistance. The eleven clinical samples tested show no genomic evidence of ML-resistance. Vigilance is needed to maintain susceptibility to heartworm preventives in regions of the world so far without resistance. The early adoption of genotyping of clinical $D$. immitis samples could provide an early indication of the potential development of ML-resistance and aid to distinguish clinical cases of heartworm infection due to $\mathrm{ML}$ resistance from those due to a lack of prevention or inadequate compliance, as has been seen in North America.

\section{Background}

Dirofilaria immitis is a veterinary parasitic filarial nematode and the cause ofdirofilariosis, a potentially fatal pulmonary infection which primarily affects canids and felids, with humans occasionally acting as an incidental host. Macrocyclic lactones (MLs) were first approved as a monthly prophylactic treatment for dirofilariosis in 1987 and remain the standard of care [1-5]. In D. immitis the MLs target and kill the infective L3 and developing L4 larvae, can reduce the fecundity of adults for up to 6 months, and help clear blood-circulating microfilariae (mf). This class of drugs has been used as an effective and generally 
safe treatment for preventing dirofilariosis. Unfortunately, complaints of heartworm preventive product ineffectiveness were brought to the US FDA Center for Veterinary Medicine (FDA/CVM) as early as 1998, only 11 years after being placed on the market [6]. In 2005, ML drug loss of efficacy (LOE) cases, documented in dirofilariosis hotspots throughout the Southern United States, were brought to public attention [6]. The heritability of ML resistant isolates was established in 2014 by experimentally infecting laboratory dogs with $D$. immitis field LOE isolates [7].

Whole genome analysis elucidated a number of single nucleotide polymorphisms (SNPs) apparently associated with a resistant phenotype [8-10]. The 10 SNPs which best differentiated the ML-resistant phenotype from the ML-susceptible phenotype were selected for analysis in clinical infections collected from the continental USA [11]. A significant correlation of the SNP loci frequencies and the ML microfilaricidal response phenotype was observed in 9 of the 10 SNPs. The clinical validation of the molecular markers for $\mathrm{ML}$ resistance in $D$. immitis provides the first genetic test to confirm the development of ML-resistant isolates which are genetically distinct from wild-type populations. These markers can be used to differentiate between ML-resistant $D$. immitis isolates versus cases of opportunistic infections.

The prevalence of Dirofilaria infections is on the rise in Europe [12]. The rise in Dirofilaria infections is likely to be the result of the increased temperatures due to climate change and the increased movement of companion animals across borders [13-15]. Hundreds of thousands of dogs are relocated internationally each year in Europe, with more than 300,000 entering the United Kingdom via the EU Pet Travel Scheme (PETS) alone [16]. Large numbers of dogs are also relocated throughout North America, with a recent report from Canada demonstrating that dogs originating in USA are positive for heartworm at double the frequency of Canadian dogs [17]. D. immitis infections were reported in 109 dogs in Austria, with the dogs originating from Hungary, Greece, the western Balkans, the Iberian Peninsula, Romania, USA, or Bulgaria [18]. As temperatures continue to rise $D$. immitis infections are expected to spread north from the Mediterranean [19]. Autochthonous transmission of canine dirofilariosis has encroached on regions previously untouched by naturally occurring infections such as Hungary and Balkan countries [20-23]. Given this geographical expansion of dirofilariosis it will be important to monitor the effectiveness of MLs in Europe and the possible emergence of resistance. To date there is no published information on the genetic make-up of European strains and the prevalence of SNPs associated with susceptibility and resistance to MLs. These insights are needed to help distinguish LOE cases caused by poor owner treatment compliance from those caused by the emergence of resistance.

\section{Methods}

\section{US Samples}

Three newly established US laboratory-maintained isolates were analyzed, two susceptible isolates, Berkeley, and Georgia II, and one resistant isolate, WildCat (Table 1) [24]. The three isolates were utilized in dose confirmation studies for the new milbemycin oxime based ML chemoprophylactic Credelio Plus ${ }^{\mathrm{Tm}}$. 
The Berkeley D. immitis isolate originated in Berkeley County, South Carolina, and had been maintained under laboratory conditions since April 2014. The Georgia II isolate originated in Vidalia, Georgia and had been maintained under laboratory conditions since April 2013. The ML resistant Wildcat isolate originated in West Liberty, Kentucky and had been maintained under laboratory conditions since August 2012. The 3 isolates were provided by TRS Lab Inc., Athens, GA, USA.

Table 1

Dirofilaria immitis sample identification, life stage, treatment history and origin for the 3 US laboratorymaintained isolates and the 11 European clinical samples which underwent MiSeq Illumina Sequencing.

\begin{tabular}{|c|c|c|c|c|c|}
\hline Sample & $\begin{array}{l}\text { Life } \\
\text { Stage }\end{array}$ & Isolate & Dog Type & $\begin{array}{l}\text { ML } \\
\text { Treatment }\end{array}$ & Origin \\
\hline \multicolumn{6}{|c|}{ US laboratory-maintained isolates } \\
\hline WildCat & Blood mf & WildCat & Unknown & Untreated & West Liberty, KY, USA \\
\hline Berkeley & Blood mf & Berkeley & Unknown & Untreated & $\begin{array}{l}\text { Berkeley County, SC, } \\
\text { USA }\end{array}$ \\
\hline Georgia & Blood mf & Georgia & Unknown & Untreated & Vidalia GA, USA \\
\hline
\end{tabular}

\section{European clinical samples}

\begin{tabular}{|c|c|c|c|c|c|}
\hline T2 & Adult $\otimes$ & Unknown & Unknown & Unknown & Lombardy Region, Italy \\
\hline T3 & Adult $\otimes$ & Unknown & Unknown & Unknown & Lombardy Region, Italy \\
\hline T4 & Adult $\otimes$ & Unknown & Unknown & Unknown & Lombardy Region, Italy \\
\hline T9 & Adult $\otimes$ & Unknown & Unknown & Unknown & Hungary \\
\hline T10 & Adult $\otimes$ & Unknown & Unknown & Unknown & Hungary \\
\hline T11 & Adult $\otimes$ & Unknown & Unknown & Unknown & Hungary \\
\hline C1 & Blood mf & Unknown & Canary Mastiff & Untreated & Canary Island, Spain \\
\hline $\mathrm{C} 2$ & Blood mf & Unknown & Canary Mastiff & Untreated & Canary Island, Spain \\
\hline C4 & Blood mf & Unknown & Canary Hound & Untreated & Canary Island, Spain \\
\hline C5 & Blood mf & Unknown & Canary Mastiff & Untreated & Canary Island, Spain \\
\hline M & Blood mf & Unknown & $\begin{array}{l}\text { Spanish } \\
\text { Greyhound }\end{array}$ & Ivermectin & $\underset{\star}{\text { Huelva, Andalusia Spain }}$ \\
\hline
\end{tabular}

\section{European Sample Details}


Eleven European clinical samples were collected from 7 different canines (Figure 1). Samples T2, T3, and T4 were collected from a single canine host in Lombardy, Italy. The dog died of unrelated causes and ML treatment history is unknown. The adult female worm samples were collected at necropsy and preserved in ethanol. Samples T9, T10, and T11 were collected from a single dog in Hungary. The ML treatment history is unknown. The adult female worm samples were collected at necropsy and preserved in ethanol. Samples C1, C2, C4, and C5 were collected from a large dog shelter on Gran Canaria, in the Canary Islands, Spain (Table 1). The dogs were delivered to the shelter and there was no history of ML prophylaxis in the documentation delivered together with the animals. They were hunting and guard dogs that lived in rural areas of the island. Traditionally, these dogs are constantly kept outdoors, without attention to sanitation, and generally these animals do not receive dirofilariosis preventive. These dogs act as a parasite reservoir, contributing to the fact that the prevalence of heartworm disease in dogs in Gran Canaria has stagnated for 15 years: the general prevalence remains at around $20 \%$ without reduction in the incidence, while in hound dogs the prevalence remains around 40-45\% [25] There are several factors that influence this: economic, low socio-cultural level and false beliefs. The dogs from which the Gran Canaria samples were taken almost certainly never received prophylaxis. Finally, sample M was collected from a Spanish Greyhound from Huelva, Andalusia, Spain adopted and relocated to Savona, Italy (Table 1). Following adoption M was placed on 24 months of monthly ivermectin at the standard dose rate without suppression of microfilariae.

\section{Sample Processing and DNA Extraction}

The canine venous blood samples of the 3 US laboratory-maintained isolates and the 5 European clinical blood samples were shipped to McGill University for immediate processing. The mf were extracted from the blood by filtration [8]. The blood was diluted 1:1 with $\mathrm{NaHCO}_{3}$ solution and passed throughpolycarbonate membrane filters ( $3.0 \mu \mathrm{m} ; 25 \mathrm{~mm}$; Sterlitech® Corporation, Kent, WA, USA) to isolate $\mathrm{mf}$. The 6 adult worms from Italy and Hungary were shipped to McGill University and rehydrated in PBS prior to genomic DNA extraction.

Genomic DNA from the mf samples and the rehydrated adult worms were extracted using the QIAamp® DNA Micro kit (Qiagen Inc., Toronto, ON). DNA concentrations were determined with the Quant-iT ${ }^{\mathrm{TM}}$ PicoGreen DNA Assay Kit (Invitrogen®, Life Technologies Inc., Burlington, ON, Canada). The 14 samples were stored at $-80^{\circ} \mathrm{C}$ prior to being sent to Génome Québec for sequencing.

\section{SNP Markers}

The 9 SNP markers used to analyse the status of ML resistance in the European samples were the top 9 markers clinically validated in 2018 to best differentiate ML-susceptible and ML-resistant phenotypes [11] (Table S1). The SNP 10 marker on nDi.2.2.scaf00597 at position 12915 was not chosen for further analysis as it was not considered a reliable indicator for susceptible versus resistant genotyping.

\section{Sequencing}


The regions encompassing the 9 SNPs of interest were sequenced on an Illumina MiSeq Platform, at a coverage of 2000X. The Fluidigm Access Array system performed target enrichment using array-based PCR amplification of the genomic target regions. The 14 samples underwent parallel amplification using custom primers with added CS1 and CS2 tails, as described in Ballesteros et al. [11] (Table S1). The samples were barcoded during target enrichment which allowed for multiplexed sequencing, and adapter sequences were added during the PCR amplification reaction.

\section{Data Analysis}

Trimmomatic was used to trim for minimal trailing quality (30 PHRED score) and filter for minimum read length by removing the Illumina sequencing adapters from read and adapter clippings [26]. The resulting read pairs were aligned to the $D$. immitis reference genome $\mathrm{nDi} .2 .2$ (http://www.nematodes.org/genomes/dirofilaria_immitis) using BWA-mem (http://bio-

bwa.sourceforge.net/) resulting in binary alignment map files (BAM) [27]. The alignments were processed with Picard (https://broadinstitute.github.io/picard) for the realignment of indels, mate fixing, and marking of duplicate reads. BVATools (https://bitbucket.org/mugqic/bvatools/src) was used to extract base frequencies at each of the 9 SNP positions and the read frequencies were assimilated to the allele frequencies (Table S2). The allele frequency variance at the 9 SNP positions were compared to the previously described allele frequencies of the ML susceptbile populations by calculating the Fixation Index $\left(F_{S T}\right)$ [8-11]. The average $F_{S T}$ of each sample was calculated for the top 2 SNP-F $F_{S T}$ values for the 3 US laboratory-maintained isolates and the 11 European clinical samples from 7 dogs. The $F_{S T}$ value is the average coefficient of inbreeding in a population and measures the difference in allele frequencies $[28,29]$. It is a measure of population differentiation due to genetic structure with values ranging from 0 to 1 . A value of 0 indicates no differentiation between subpopulations, whereas a value of 1 indicates complete differentiation. For the purpose of considering a population of microfilariae from a dog, we consider frequencies of over 0.15 at several of the indicator SNPs as likely showing a resistant population in the dog, while frequencies of the alternative allele at several SNPs of $>0.05$ to 0.15 may be borderline or suggesting an early stage in the selection for resistance.

\section{Results}

\section{$\mathrm{F}_{\mathrm{ST}}$ Genotyping}

DNA from the 3 US and 11 European $D$. immitis samples were sent to Génome Québec and underwent MiSeq Illumina Sequencing. The regions surrounding the 9 SNP markers of interest were aligned to the $D$. immitis reference genome $\mathrm{nDi}$.2.2. A 2-SNP pairwise $\mathrm{F}_{\mathrm{ST}}$ model was used to estimate an ML-susceptibility profile in comparison to the earlier characterized susceptible profile (Table 2). The 2 SNP markers utilized are located on nDi.2.2.scaf00046 at positions 22857 and 76278; herein referred to as SNP 4 and SNP 5, respectively. The highly predictive 2-SNP pairwise $\mathrm{F}_{\mathrm{ST}}$ model utilized in this study was determined via ROC curve performance as previously described [11]. 
Table 2

Calculated 2-SNP model pairwise $\mathrm{F}_{\mathrm{ST}}$ of SNP 4 and SNP 5 previously described (Ballesteros et al. [11]) and predicted profile of the 3 US laboratory-maintained isolates and the 11 European clinical samples.

\begin{tabular}{|lll|}
\hline Sample ID & \multicolumn{1}{|l|}{$\mathbf{F}_{\text {ST }}$ (Sample vs SUS profile) } & Profile predicted based on Genotype \\
\hline US laboratory-maintained isolates & \\
\hline WildCat & 0.33 & Resistant \\
\hline Berkeley & 0.00 & Susceptible \\
\hline Georgia II & 0.07 & Susceptible \\
\hline European clinical samples & \\
\hline T2 & 0.00 & Susceptible \\
\hline T3 & 0.00 & Susceptible \\
\hline T4 & 0.00 & Susceptible \\
\hline T9 & 0.00 & Susceptible \\
\hline T10 & 0.00 & Susceptible \\
\hline T11 & 0.00 & Susceptible \\
\hline C1 & 0.00 & Susceptible \\
\hline C2 & 0.00 & Susceptible \\
\hline C4 & 0.00 & Susceptible \\
\hline C5 & 0.00 & Susceptible \\
\hline M & 0.00 & Susceptible \\
\hline
\end{tabular}

The laboratory-maintained resistant isolate WildCat had a 2-SNP model pairwise $F_{S T}$ of 0.33 calculated based on SNP 4 and SNP 5, which signals a deviation from the wildtype (susceptible) genome. Georgia II displayed minor deviation from the reference genome with a 2-SNP model pairwise $F_{S T}$ of 0.07 . Whereas, Berkeley had a fixation index of 0.00 which denotes no differentiation from the wildtype genotype. The pairwise 2-SNP model $F_{S T}$ values of the 11 European clinical samples were 0.00 (Table 2). Similarly, the 11 European samples from 7 dogs also displayed a pairwise $F_{S T}$ of 0.00 at the other 7 SNP loci. It indicates that the European samples resemble the US ML-susceptible isolates and have genotypes consistent with susceptibility as defined by the SNP molecular markers.

The three adult female worm samples from Lombardy, Italy: T2, T3, and T4, were all collected from the same canine host. The three samples all presented a consistent 2-SNP pairwise $F_{S T}$ genotype between 
the worms collected from the same host (Dataset S1). The same phenomenon was seen in the three adult female worms samples T9, T10, and T11, which were collected from one canine host in Hungary. A mixed genotype Dirofilaria infection does not appear to be occuring in the two canine hosts from the Lombardy region of Italy, or from Hungary.

\section{Alternate Allele Frequency}

The allele frequencies were calculated from the read frequencies using BVAtools in comparision to the reference genome nDi.2.2 (Table S2). The average alternative allele frequency for each of the 9 SNP positions was then plotted for the three US laboratory-maintained isolates and the European sample $\mathrm{M}$ (Figure 2). $\mathrm{M}$ acts as a representative for the 11 European samples. Compared to all other samples, WildCat carried higher frequencies of the alternate nucleotide for all SNP markers, with a genotype comparable to other known ML-resistant laboratory-maintained isolates, such as JYD-34 [9].

The Berkeley isolate displayed some small variation in comparison to the reference genome at SNPs 2, 7 and 9. The Georgia II isolate displayed a low level of variation from 5 to $14 \%$ at SNPs $2,4,5,6$, and 9 . The $\mathrm{M}$ sample and all other European samples displayed no alternate allele frequency and had genotypes consistent with ML-susceptibility and the $\mathrm{nDi}$.2.2. reference genome The $\mathrm{M}$ sample had a genotype most aligned to the known ML susceptible Missouri isolate (Figure 2). SNP 9 showed the least differentiation between all the USA samples.

\section{Discussion}

Previous research indicates an increase in D. immitis infections in the USA [30, 31]. ML-resistant isolates, genetically distinct from the wildtype population, have been confirmed and documented in the southern US during the last decade. This is perhaps not unexpected after long term and widespread use of prophylactic MLs [7-11]. SNP analysis has been conducted on previously established US laboratorymaintained isolates with known phenotypes such as the ML-susceptible Missouri and Kentucky isolates, as well as the ML-resistant JYD-34 and Metairie isolates [9]. The current study is the first to complete genomic level analysis of the newly established ML-susceptible isolates Berkeley and Georgia II, and the ML-resistant isolate WildCat [24].

The research of Ballesteros et al. [11] demonstrated that the most highly predictive SNP pairwise $\mathrm{F}_{\mathrm{ST}}$ combination was that of SNP 4 and 5; compared to the original 10 SNP markers. The 2-SNP pairwise $F_{\text {ST }}$ model correctly differentiated all samples in the Ballesteros et al. study. Using the 9 most effective markers, our data illustrated the same phenomenon. The 2-SNP pairwise $F_{S T}$ better differentiated the US laboratory-maintained samples. The European clinical samples were so closely aligned to the reference genome both predictive models functioned comparably. When reviewing the alternate allele frequencies, SNPs 4, 5, and 6 demonstrated the highest levels of alternate allele variability between the ML-susceptible Berkeley and Georgia II isolates, and the European sample M when compared with the ML-resistant 
WildCat isolate (Figure 2). These results are consistent with previously published clinical validation data [11]. SNP 9 does not differentiate the populations as well as the other SNPs.

The Berkeley isolate displays a susceptible genotype consistent with that of the other previously categorized ML-susceptible isolates at the $9 \mathrm{SNP}$ markers in comparison with the $D$. immitis reference genome nDi.2.2. The ML-resistant WildCat isolate has a high degree of variability across all 9 SNP markers. It has a 2-SNP pairwise $\mathrm{F}_{\mathrm{ST}}$ of 0.33 . WildCat presents an alternate allele frequency of greater than $20 \%$ at all 9 SNP markers, and greater than $39 \%$ at SNP markers 1-7. Georgia II displays both an alternate allele frequency and 2-SNP pairwise $\mathrm{F}_{\mathrm{ST}}$ between Berkeley and WildCat isolates. Georgia II has a 2-SNP pairwise $F_{S T}$ of 0.07 . The Georgia II isolate had variability at SNPs $2,4,5,6$, and 9, which ranged from 5 to $14 \%$. The variability seen in this isolate across multiple SNP markers was higher compared to previously published susceptible isolates, Berkeley, and the European clinical samples examined in this study (Figure 2).

When clinical trials are run in vivo, only one dose rate is usually tested; that proposed for the commercial product. Therefore, it is not easy to pick up early evidence of resistance selection. The genomic variability of the Georgia II isolate compared to the Berkeley isolate and other known ML-susceptible isolates raises several questions. To date US laboratory-maintained isolates are characterized as ML susceptible due to their elimination at the commercial dose rate of treatment or proposed lack of ML-drug exposure. A lack of history of prophylactics in a particular dog does not necessarily mean that the ancestors of the challenge worms had not been exposed to repeated ML chemoprophylaxis. In fact, the AHS recommendation that all dogs in the USA be treated 12 months of the year means that $D$. immitis, in the USA, whose ancestors were truly naïve to ML prophylaxis, are probably becoming rare.

Genomic level testing, via the SNP molecular markers, provides key background information on the $D$. immitis isolates currently being used in laboratory and pharmaceutical research. However, little information has been documented on the potential development of ML-resistance in European D. immitis populations. The European $\mathrm{M}$ sample, a representative for the 11 European samples, had the genotype and allele frequencies most closely aligned with the $D$. immitis reference genome nDi.2.2 (Figure 2). The genotype analysis of the European clinical samples showed that all 11 samples had genotypes consistent with susceptibility as defined by the molecular marker 2-SNP model pairwise $F_{S T}$. While only a small number of $D$. immitis samples were genotyped, from a limited number of countries, the results of the study indicate no evidence for the development of ML-resistance in Europe or its introduction via movement of infected animals. This conclusion is supported by data presented at the 6 th Congress of the European Society of Dirofilariosis and Angiostrongylosis in Belgrade, in 2018 [32]. A larger study across more geographical locations should be considered for conclusive evidence.

\section{Conclusion}

Autochthonous transmission of canine $D$. immitis appear to be migrating from the Mediterranean and Iberian Peninsula moving further into Central and Northern Europe [13-15, 19], likely, a result of the 
movement of dogs and cats around Europe, and possibly a result of increasing mosquito populations. To date, no case of ML-resistant $D$. immitis infection has been documented in Europe. As the number of $D$. immitis infections continues to rise and spread throughout Europe, the early adoption of genotyping of clinical $D$. immitis samples could provide an early indication of the potential development of MLresistance and aid to distinguish clinical cases of heartworm infection due to $\mathrm{ML}$ resistance from those due to a lack of prevention or inadequate compliance, as has been seen in North America.

Epidemiological surveys of $D$. immitis samples collected across Europe from historically endemic regions of the Mediterranean, newly endemic regions such as the Balkans and Austria, and previously nonendemic regions of Northern Europe can provide insight into the genetic makeup and genetic diversity of European clinical samples $[18,20,21]$. The 2-SNP model pairwise $F_{S T}$ genotypic analysis of clinical $D$. immitis samples can be utilized to help monitor the efficacy and susceptibility of ML-based heartworm preventives.

\section{Abbreviations}

ML: Macrocyclic Lactone; mf: Microfilaria; SNP: Single nucleotide polymorphism; PETS: EU Pet Travel Scheme; FDA/CVM: US Food and Drug Administration / Center for Veterinary Medicine; LOE: Loss of efficacy; BAM: Binary alignment map files.

\section{Declarations}

\section{Ethics approval and consent to participate}

All experimental procedures were approved by McGill University in accordance with relevant guidelines and regulations.

\section{Consent for publication}

Elanco consents to release of this information for publication.

\section{Availability of data and materials}

The data supporting the conclusions of this article are included within the article. Bioinformatic files can be provided by authors upon reasonable request.

\section{Competing interests}

The research was supported by Elanco Animal Health. LY and HS are employees of the sponsor. However, the sponsor of the research exercised no influence over the conduct of the research. The other authors declare no conflicts of interest that could influence the conduct or results of this study.

\section{Funding}


This work was supported by Elanco Animal Health (Greenfield, IN, USA).

\section{Authors' contributions}

Experiments were conceived and designed by LY and RKP. D. immitis samples were provided by DT, EC, LK, and HS. Experiments and data analysis were performed by EC*. The manuscript was written by EC* and RKP. All authors read and approved the final manuscript.

\section{Acknowledgements}

We thank Génome Québec Innovation Centre for sequencing the samples on their MiSeq platform and preparation of the alignment files. We thank TRS Lab Inc. for providing the US D. immitis isolates. We thank Compute Canada and Calcul Québec for use of the Beluga server for data analysis. We thank Ms. Kathy Keller for her help and in sample preparation and submission. We also thank Dr. Cristina Ballesteros for help and guidance in the analysis of the sequencing data. DT thanks Prof. Antonio Frangipane di Regalbono for the help in recovering the Italian Dirofilaria immitis specimens. LK thanks Luigi Venco for reporting the $\mathrm{M}$ sample as a possible resistant.

\section{References}

1. Chabala, JC, Mrozik H, Tolman RL, Eskola P, Lusi A, Peterson LH, et al. Ivermectin, a new broadspectrum antiparasitic agent. J. Med. Chem. 1980; 23(10): 1134-1136.

2. Campbell, WC. An introduction to the avermectins. N Z Vet J 1981; 29(10): 174-178.

3. Campbell, WC, Fisher MH, Stapley EO, Albers-Schönberg G, Jacob TA. Ivermectin: a potent new antiparasitic agent. Science. 1983; 221(4613): 823-828.

4. Ohishi I, Katae H, Hayasaki M, Nakagaki K, Tada Y. Prophylactic activity of ivermectin against Dirofilaria immitis infection in dogs : Establishment of effective dose and administration schedule. Jpn. J. Vet. 1987; 49(3): 439-445.

5. Ohishi I, Katae H, Hayasaki M, Tada Y. Prophylactic activity of ivermectin against Dirofilaria immitis infection in dogs: Larvicidal activity of ivermectin against $D$. immitis larvae 30 days after infection. Jpn. J. Vet. 1987; 49(1): 115-120.

6. Hampshire VA. Evaluation of efficacy of heartworm preventive products at the FDA. Vet. Parasitol. 2005; 133(2): 191-195.

7. Pulaski CN, Malone JB, Bourguinat C, Prichard R, Geary T, Ward D, et al. Establishment of macrocyclic lactone resistant Dirofilaria immitis isolates in experimentally infected laboratory dogs. Parasites Vectors. 2014; 7(1): 494.

8. Bourguinat, C, Lee ACY, Lizundia R, Blagburn BL, Liotta JL, Kraus MS, et al. Macrocyclic lactone resistance in Dirofilaria immitis: Failure of heartworm preventives and investigation of genetic markers for resistance. Vet Parsitol. 2015; 210(3): 167-178. 
9. Bourguinat, C, Keller K, Xia J, Lepage P, McTier TL, Woods DJ, Prichard RK. Genetic profiles of ten Dirofilaria immitis isolates susceptible or resistant to macrocyclic lactone heartworm preventives. Parasites Vectors. 2017; 10(Suppl 2): 504.

10. Bourguinat, C, Lefebvre F, Sandoval J, Bondesen B, Moreno Y, Prichard RK. Dirofilaria immitis JYD-34 isolate: whole genome analysis. Parasites Vectors. 2017; 10(Suppl 2): 494.

11. Ballesteros C, Pulaski CN, Bourguinat C, Keller K, Prichard RK, Geary TG. Clinical validation of molecular markers of macrocyclic lactone resistance in Dirofilaria immitis. Int J Parasitol Drugs Drug Resist 2018. 8(3): 596-606.

12. Genchi C, Kramer LH. The prevalence of Dirofilaria immitis and D. repens in the Old World. Vet Parasitol. 2020; 280: 108995.

13. Genchi C, Rinaldi L, Cascone C, Mortarino M, Cringoli G. Is heartworm disease really spreading in Europe? Vet Parasitol. 2005; 133(2): 137-148.

14. Genchi, C, Rinaldi L, Mortarino M, Genchi M, Cringoli C. Climate and Dirofilaria infection in Europe. Vet Parasitol. 2009; 163(4): 286-292.

15. Traversa D, Di Cesare A, Conboy G. Canine and feline cardiopulmonary parasitic nematodes in Europe: emerging and underestimated. Parasites Vectors. 2010; 3(1): 62.

16. Norman C, Stavisky J, Westgarth C. Importing rescue dogs into the UK: reasons, methods and welfare considerations. Vet Rec. 2020; 186(8): 248.

17. Jacobson LS, Ward KA, Lacaden AB, Hornak TA. Prevalence of heartworm in relocated, local and outreach clinic dogs: A Canadian sheltering perspective. Vet Parasitol. 2020; 283:109081.

18. Sonnberger K, Duscher GG, Fuehrer H-P, Leschnik M. (2020). Current trends in canine dirofilariosis in Austria-do we face a pre-endemic status? Parasitol 2020; 119(3): 1001-1009.

19. Montoya-Alonso JA, Carretón E, Morchón R, Silveira-Viera L, Falcón Y,Simón F. The impact of the climate on the epidemiology of Dirofilaria immitis in the pet population of the Canary Islands. Vet Parasitol. 2016; 216: 66-71.

20. Bacsadi Á, Papp A, Szeredi L, Tóth G, Nemes C, Imre V, et al. Retrospective study on the distribution of Dirofilaria immitis in dogs in Hungary. Vet Parasitol. 2016; 220: 83-86.

21. Ionică AM, Matei IA, D’Amico G, Ababii J, Daskalaki AA, Sándor AD, et al. Filarioid infections in wild carnivores: a multispecies survey in Romania. Parasites Vectors. 2017; 10(1): 332.

22. Farkas R, Mag V, Gyurkovszky M, Takács N, Vörös K, Solymosi N. The current situation of canine dirofilariosis in Hungary. Parasitol. 2020;119(1): 129-135.

23. Savić S, Stosic MZ, Marcic D, Hernández I, Potkonjak A, Otasevic S, et al. Seroepidemiological Study of Canine and Human Dirofilariasis in the Endemic Region of Northern Serbia. Front Vet Sci. 2020; 7(571).

24. Elanco GmbH. Credelio Plus. EMEA/V/C/005325/0000. European Medicines Agency. 2021.

25. Montoya-Alonso JA, Carretón E, Juste MC, Mellado I, Morchón R, Simón F. Epidemiological survey of canine heartworm disease on the island of Gran Canaria (Canary Islands - Spain) between 2000 and 
2008. Vet Parasitol. 2010 Oct 11;173(1-2):165-8.

26. Bolger AM, Lohse M, Usadel B. Trimmomatic: a flexible trimmer for Illumina sequence data. BOINFP. 2014; 30(15): 2114-2120.

27. Li H, Durbin R. Fast and accurate short read alignment with Burrows-Wheeler transform. BOINFP. 2009; 25(14): 1754-1760.

28. Kitada S, Kitakado T, Kishino H. Empirical Bayes Inference of Pairwise FST and Its Distribution in the Genome. Genetics 2007; 177(2): 861-873.

29. Holsinger KE, Weir BS. Genetics in geographically structured populations: defining, estimating and interpreting FST. Nat Rev Genet. 2009; 10(9): 639-650.

30. American Heartworm Society, (2020). "Heartworm Incidence Maps" https://www.heartwormsociety.org/veterinary-resources/incidence-maps Accessed April 29th, 2021.

31. Drake J, Wiseman S. Increasing incidence of Dirofilaria immitis in dogs in USA with focus on the southeast region 2013-2016. Parasites Vectors. 2018;11(1):1-7

32. Diakou A, Koutinas C, Bourguinat C, Ballesteros C, Dimzas D, Chalkias V, Batra M, Traversa D, Prichard R. Heartworm infection in military dogs under preventive treatment: Questions and suggested answers. 2018; 6th European Dirofilaria and Angiostrongylus Days, Belgrade.

\section{Figures}




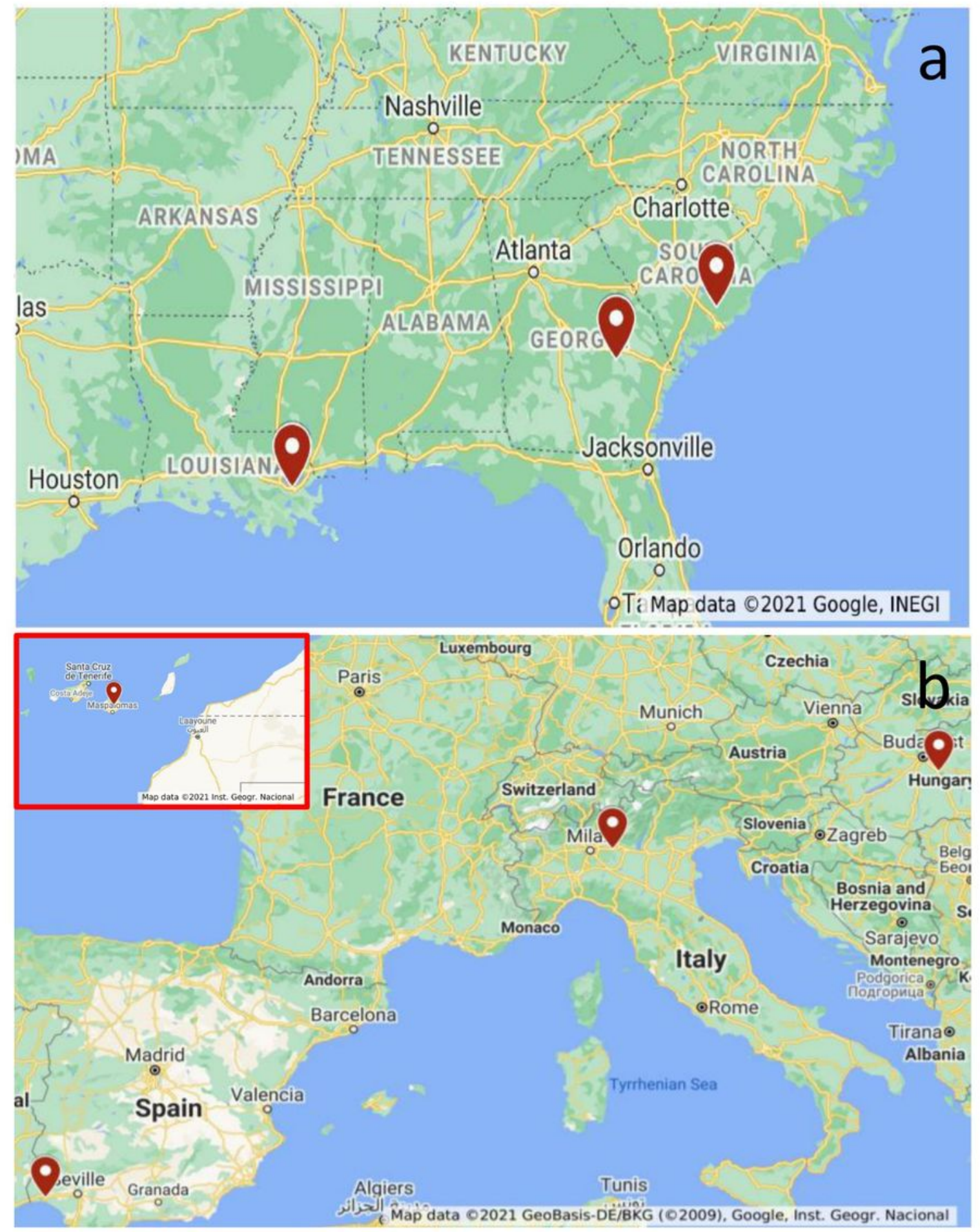

\section{Figure 1}

a) Distribution of US laboratory-maintained isolates received from TRS Laboratories b) Distribution of European samples received from Italy, Hungary, Spain, and the Canary Islands. The maps were created using Google Maps, accessed May $2^{\text {nd }}$ and August $26^{\text {th }}, 2021$. 


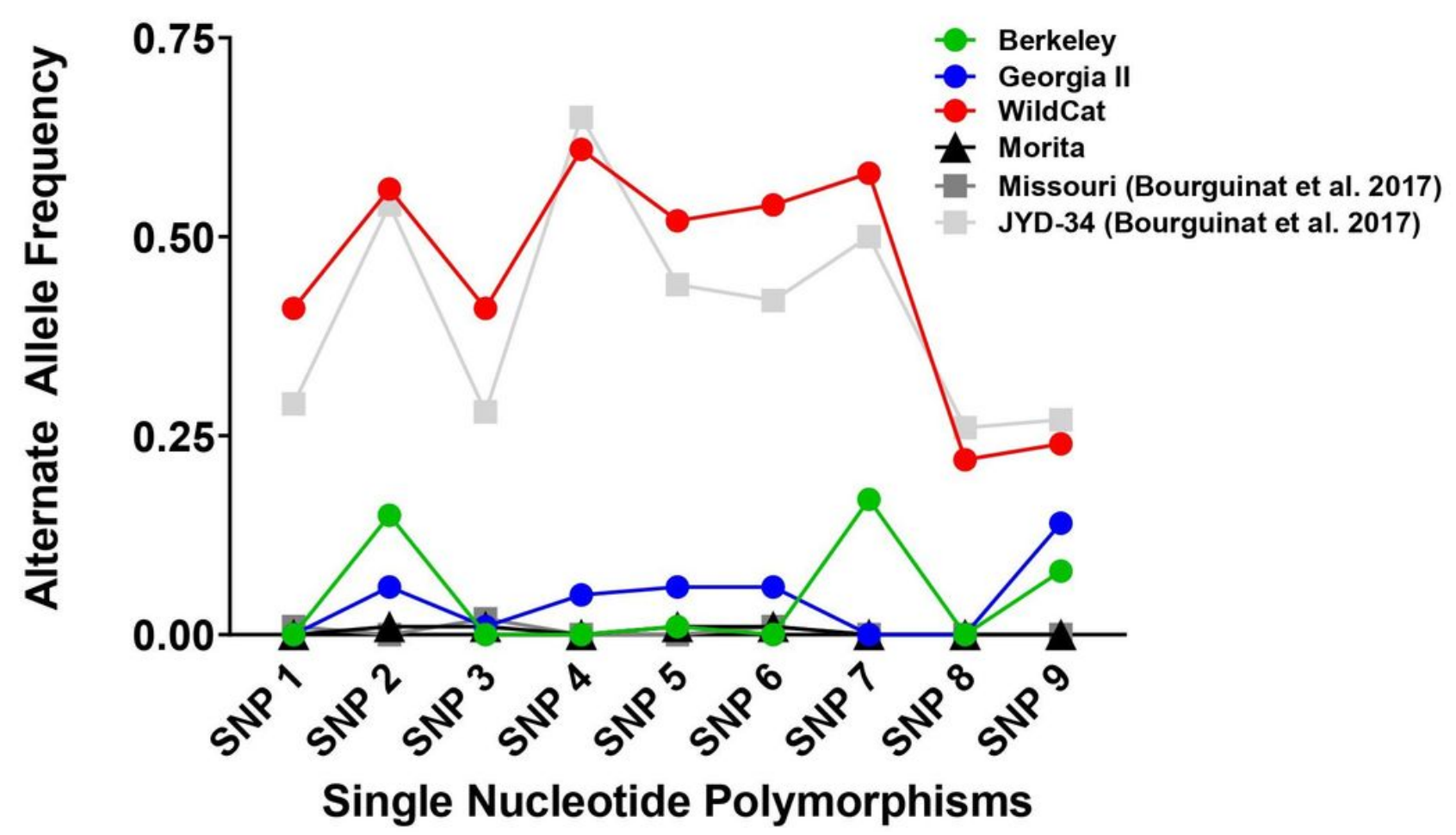

Figure 2

The alternate nucleotide frequency of the 9 molecular markers, in sequential scaffold order, for the Berkeley isolate (Green @), Georgia II isolate (Blue @), WildCat isolate (Red $\bullet$ ), and the European M sample (Black $\boldsymbol{\Lambda}$ ) compared to the known ML-susceptible Missouri isolate (Dark Grey $\boldsymbol{\square}$ ), and the known ML-resistant isolate JYD-34 (Light Grey $\boldsymbol{\square}$ ). The alternative nucleotide frequencies for the 9 SNPs molecular markers were prepared in comparision to the $D$. immitis reference genome nDi.2.2.

\section{Supplementary Files}

This is a list of supplementary files associated with this preprint. Click to download.

- CURRYEmilyetalTableS110012022.pdf

- CURRYEmilyetalDataSetS110012022.pdf

- CURRYEmilyetalGraphicalAbstract10012022.pdf 